\title{
Repair of Inaccessible Ventral Dural Defect in Thoracic Spine: Double Layered Duraplasty
}

\author{
Dong-Hyun Lee, Kyoung-Tae Kim, Jeong-III Park, Ki-Su Park, Dae-Chul Cho, Joo-Kyung Sung \\ Department of Neurosurgery, Spine Center, Kyungpook National University Hospital, \\ Kyungpook National University College of Medicine, Daegu, Korea
}

We propose a double layered (intradural and epidural patch) duraplasty that utilizes Lyoplant and Duraseal. We examined a 47-year-old woman after decompression for thoracic ossification of posterior longitudinal ligament was performed in another hospital. On postoperative day 7, she complained of weakness in both legs. Postoperative magnetic resonance imaging (MRI) showed cerebrospinal fluid (CSF) collection with cord compression. In the operative field, we found 2 large dural defects on the ventral dura mater. We performed a conventional fat graft with fibrin glue. However, the patient exhibited neurologic deterioration, and a postoperative MRI again showed CSF collection. We performed dorsal midline durotomy and inserted a intradural and epidural Lyoplant patch. She immediately experienced diminishing back pain postoperatively. Her visual analog scale and motor power improved markedly. Postoperative MRIs performed at 2 and 16 months showed no spinal cord compression or CSF leakage to the epidural space. We describe a new technique for double layered duraplasty. Although we do not recommend this technique for all dural repairs, double-layered duraplasty may be useful for repairing large inaccessible dural tears in cases of persistent CSF leakage refractory to conventional management.

Key Words: Dura mater $\cdot$ Repair $\cdot$ Microtomy $\cdot$ Cerebrospinal fluid leak

\section{INTRODUCTION}

Many spine surgeons occasionally encounter a patient with an undesirable dural tear accompanied by cerebrospinal fluid (CSF) leakage, especially when performing surgery for ossification of the posterior longitudinal ligament (OPLL) and/or revision surgery. CSF leakage can induce back pain, nerve injury, intracranial hypotension syndrome, delayed wound healing, and/or wound infection ${ }^{1,3,8,12,17)}$. Therefore, the management of a dural tear and CSF leakage is very important in determining the outcome of patients.

The best method to manage dural tears and CSF leakage is primary dural sutures $3,4,6,7,11)$. However, primary dural sutures can be impossible if the location of the dural tear is inaccessible or the size of the defect is large. In such cases, other operative techniques, such as artificial dural grafts, autologous fat grafts, and fascia grafts supplemented with fibrin glue, have been recommended ${ }^{2-5,9,18,19)}$. However, if these methods fail

- Received: March 1, 2016 • Revised: May 29, 2016

- Accepted: May 30, 2016

Corresponding Author: Kyoung-Tae Kim

Department of Neurosurgery, Kyungpook National University Hospital, Kyungpook National University College of Medicine, 130 Dongdukro, Jung-gu, Daegu 41944, Korea

Tel: +82-53-420-5657, Fax: +82-53-423-0504

E-mail: nskimkt7@gmail.com

$\otimes$ This is an open access article distributed under the terms of the Creative Commons Attribution Non-Commercial License (http://creativecommons.org/licenses/by-nc/4.0/) which permits unrestricted non-commercial use, distribution, and reproduction in any medium, provided the original work is properly cited. or are not amenable, other management options are required. This report is a technical proposal describing our experience with a previously unreported application of intradural and epidural patch technique by using lyophilized dura mater (Lyoplant, B. Braun Melsungen AG, Melsungen, Germany) and polyethyleneglycol hydrogel sealant (Duraseal, Integra LifeSciences Corporation, Plainsboro, NJ, USA).

\section{CASE REPORT}

A 47-year-old woman was transferred to Kyungpook National University Hospital after decompressive surgery at another hospital. A preoperative spinal computerized tomography scan and magnetic resonance imaging (MRI) showed multilevel continuous-type OPLL that extended from the inferior body of T4 to the inferior body of T7 (Fig. 1). She had experienced progressive paraparesis for 8 months. Her visual analog scale score for the dysesthesia of both legs was 8 out of 10 at presentation. A decompressive lamino-facetectomy on T5-T6 and T6-T7 and bilateral OPLL removal with pedicle screw fixation on T4, T5, T8, and T9 were performed at another hospital. On postoperative day 7 , she complained of weakness in both legs (motor power, 1/5) and severe leg pain. Postoperative MRI showed CSF collection with cord compression, and she was transferred to our hospital. We decided to perform revision surgery. In the operative field, we found 2 large dural defects on the ventral portion of the dura mater. We could not perform a primary suture, and, thus, we performed a fat graft on the epidural space of the dural defect and sprayed Duraseal 


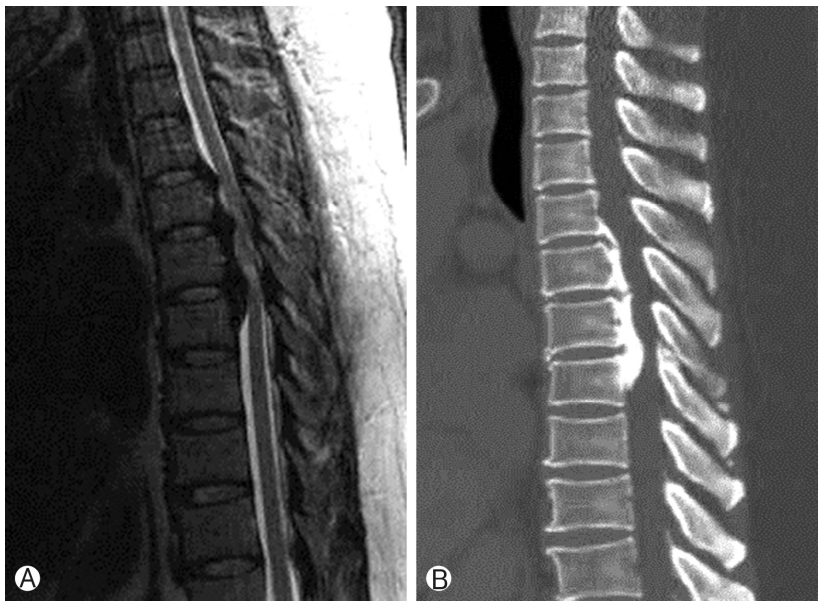

Fig. 1. Initial image. T2-weighted sagittal magnetic resonance imaging (A) and sagittal computed tomography scan (B) showed ossification of the posterior longitudinal ligament from T4 to T7.
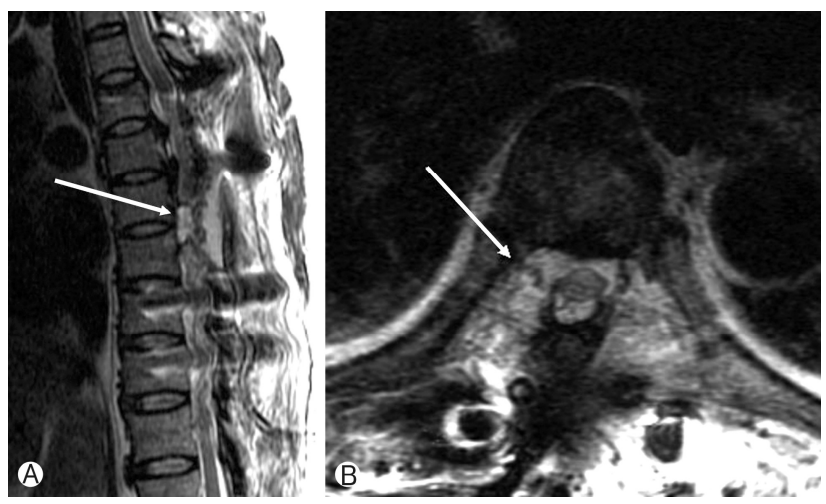

Fig. 2. Magnetic resonance imaging (MRI) after decompressive surgery for ossification of the posterior longitudinal ligament and conventional fat graft. Sagittal (A) and axial T2-weighted MRI (B) showed cerebrospinal fluid collection (arrow) with cord compression.

around the fat graft. Additionally, we inserted a hemovac in the epiduralspace and performed subarchnoid lumbar drainage on L3-4. The paraparesis improved to grades 2 (right) and 4 (left) immediately following the operation.

On the fifth day after the revision surgery, we cramped the hemovac valve and the lumbar drainage valve at the same time. Twelve hours after the cramping, she exhibited worsening paraparesis. We performed a spinal MRI, and the CSF collection with spinal cord compression reappeared (Fig. 2). We released the valve of the hemovac and the lumbar drainage, and the paraparesis then improved. Thus, we had to devise for a new method to prevent the CSF leakage arising from the large and inaccessible ventral dural defect.

We performed a midline skin incision along the pre-existing wound and carefully dissected the scar tissue around the dural mater. After an additional laminectomy of T8, normal dura
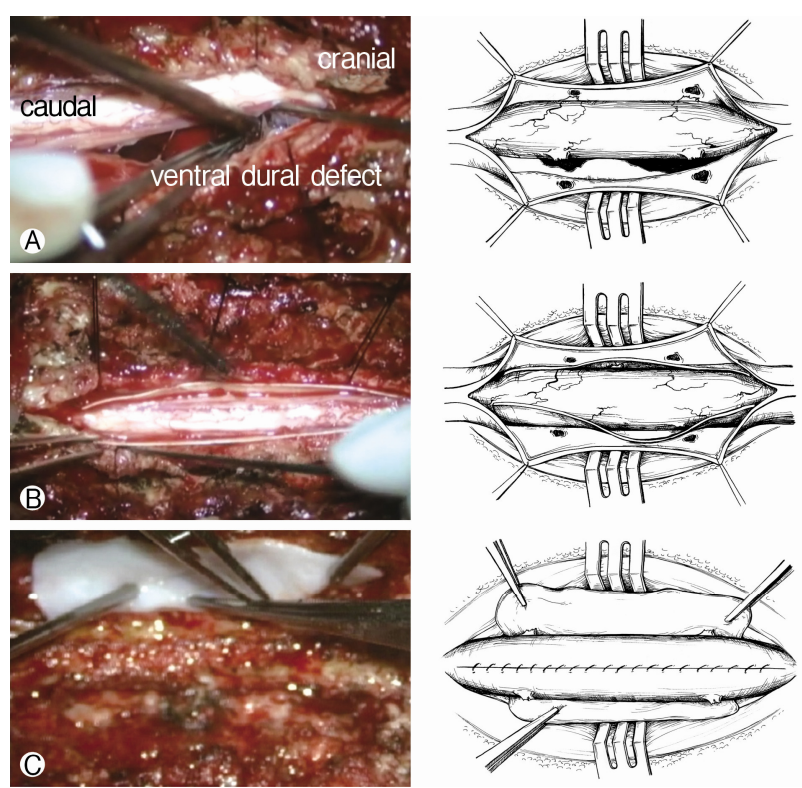

Fig. 3. (A) Intraoperative video capture image and illustration show 2 large ventral dural defects. Panels $\mathbf{B}$ and $\mathrm{C}$ show steps of procedure: $(\mathrm{B})$ orsal midline durotomy and cutting of nerve roots, (C) intradural Lyoplant (B. Braun Melsungen AG, Melsungen, Germany) patch and extradural Lyoplant patch following suturing of midline durotomy.

was observed. A dorsal midline dural incision was performed from the T4 upper vertebral body to the T8 lower vertebral body, and dentate ligaments were removed bilaterally from T5 to T7. The ventral aspect of the dura mater could be seen after cutting the T6 and T7 nerve roots. There were 2 ventral dural defects, which were $25 \mathrm{~mm} \times 10 \mathrm{~mm}$ at T5-6 and $20 \mathrm{~mm} \times$ $10 \mathrm{~mm}$ at T6-7 (Fig. 3A). We cut enough Lyoplant to cover the entire dural defects, and a dorsal midline durotomy was then performed in order to insert the subdural Lyoplant patch. The patch was inserted into the subdural space (Fig. 3B). After the patch was fixed with anchoring sutures at its margin and closed in a routine manner with 8-0 nylon. A Lyoplant patch of the same size was inserted between the outer dura mater and vertebral body (Fig. 3C), and the outside of the dural tears were fully covered. Duraseal was sprayed following the fat graft around the margin of the dural tears, and a subarachnoid lumbar drain was then inserted after wound closure layer by layer.

The total operation time was 300 minutes, and the patient tolerated the procedure well. The patient was kept on bed rest for 1 week after the operation, at which time the lumbar drain was removed and she restarted her rehabilitation program.

She experienced diminishing back pain immediately following the operation. Her visual analog scale decreased from 5 of 10 preoperatively to 2 of 10 postoperatively. Her motor power increased from 1 of $5 / 2$ of 5 (right leg/left leg) to 3 of $5 / 4$ of 5 at the 6 -week follow-up. Postoperative MRI performed at 2 months demonstrated CSF accumulation in the epidural space, but there was no spinal cord compression or 


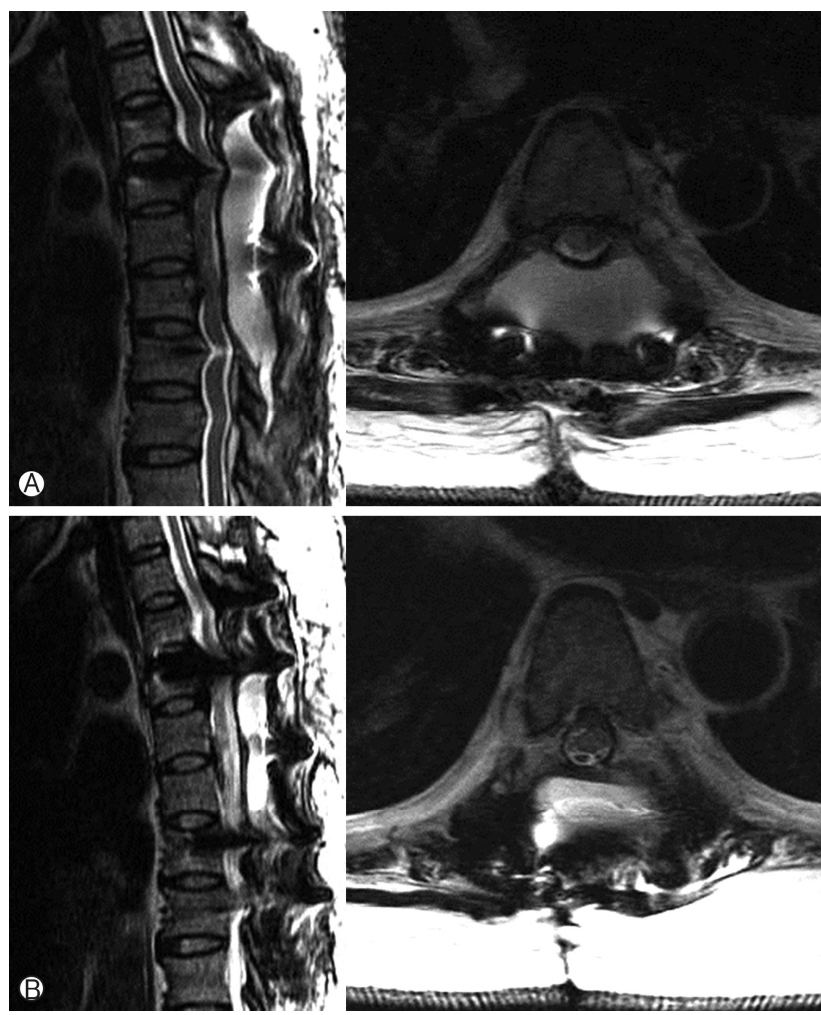

Fig. 4. (A) Postpoerative T2-weighted magnetic resonance imaging (MRI) at 2-month follow-up showed cerebrospinal fluid (CSF) accumulation in the epidural space with no spinal cord compression. (B) Postpoerative T2-weighted MRI at 16-month follow-up showed resolution of the CSF accumulation.

CSF leak (Fig. 4A). Postoperative MRI performed at 16 months presented resolution of the CSF accumulation and no CSF fistula (Fig. 4B). There were no wound complications or recurrence of symptoms through the 16 months of follow-up.

\section{DISCUSSION}

The incidence of dural tears varies from $1 \%$ to $17 \%$ according to the anatomic site, patient age, surgical approaches, and concomitant pathologies, 3,6,14,20). Dural tears could lead to the formation of a pseudomeningocele or CSF fistula, which may cause meningitis, arachnoiditis, or delayed wound healing. When a dural tear is detected intraoperatively, a watertight primary suture is mandatory ${ }^{6,10,15,20)}$. If a meticulous, watertight primary suture is impossible, other modes of repair need to be considered. A dural tear that occurs more laterally or ventrally, which is inaccessible or which does not enable approximation of the edges, is more challenging. An easily accessible dura tear that cannot be primarily sutured may be amenable to variable grafts. Autologous fascia, fat, paravertebral muscles, or dural substitutes may be applied and sutured over the defect, allowing for a good watertight seal ${ }^{6}$.

In the case of an inaccessible tear, Narotam et al. ${ }^{16}$ achieved
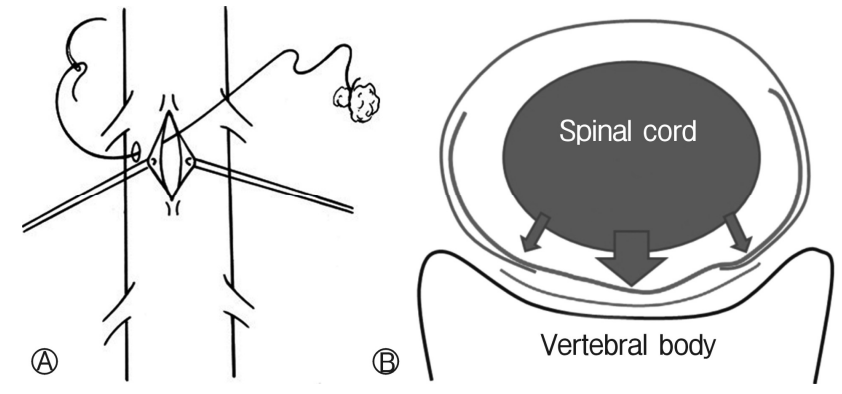

Fig. 5. (A, B) Cross section view of double-layer duraplasty.

successful results with a primary dural repair using a sutureless collagen matrix onlay graft. The collagen matrix onlay graft is placed over the dural tear, and it attaches with surface tension to the dura, where it allows a low-pressure absorptive surface to diffuse any CSF. The collagen is immediately hemostatic, initiating clot formation and resulting in a chemical seal. In addition, the collagen matrix provides a chemical signal for fibroblast infiltration. The fibroblasts use the pores in the collagen matrix as a scaffold, and they deposit new collagen, thereby reconstituting new dura. However, this technique is used for low-pressure CSF leaks, such as small dural rents and pinhole tears involving the nerve root sleeves or lateral dura. The collagen matrix attaches only through surface tension, and it therefore may not be amenable to a highpressure CSF leak, such as that arising from large ventrally located dural defects. Mayfield and Kurokawa ${ }^{13)}$ have described an intradural fat patch technique. The dura is opened at an accessible medial point, and a plug of fat that has been anchored by a string is inserted into the thecal sac and pulled through the new opening from the inside. A durotomy made in the dorsal medial dura in order to insert the fat graft is then closed with a routine watertight primary suture. Although autologous fat or muscle grafts prevent foreign body reactions ${ }^{2)}$, they have drawbacks, including difficulty in providing a watertight closure, the formation of scar tissue, and limited graft material. Because of the restricted availability of a sufficient amount of graft material and the expected mass effect of an intradural fat graft, this technique is available for small dural rents, but it is not amenable to large dural defects or multiple small dural defects.

We propose a new modified surgical technique for large dural defects or multiple dural rents when they occur in inaccessible areas. One-layered outer grafts seem to not be resistant to the pressure of a CSF leak. If the inner layer patch is added and the pressure increases, the patch strongly adheres to the dura mater (Fig. 5A). Hence, this method will provide a more watertight dural repair than a one-layer dural graft. However, a double-layer duraplasty has several anticipated problems, including stenosis of the dural sac, which is caused by inserting Lyoplant into the subdural space, and compression of the spinal cord, which is caused by buckled Lyoplant. In order to prevent these side effects, the size of the graft materi- 
al is very important. An exact size prevents stenosis and buckling of the graft material.

In order to entirely wrap a large dural defect, we cut the T6 and $\mathrm{T} 7$ nerve root. The patient complained of numbness at the T6 and $\mathrm{T} 7$ sensory dermatomes after the surgery. However, the numbness progressively subsided after medication with gabapentin, and she stopped the medication 6 months after surgery. However, in order to prevent limb weakness, the procedure of cutting the nerve roots is not possible in cases with cervical or lumbar lesions. To repair ventral dural defects at the cervical or lumbar level with this technique, it is necessary to cut the Lyoplant to a proper size to cover the ventral aspect of the dura (Fig. 5B).

\section{CONCLUSION}

In the repair of incidental dural tears, a primary watertight closure is the most preferable method. If a dural tear arises from an inaccessible area, an alternative method is required. Although we do not recommend this technique for all dural repairs that occur in this area, a double-layer duraplasty may be useful to repair an unapproachable large dural tear in cases of persistent CSF leakage refractory to conventional management.

\section{CONFLCT OF INTEREST}

No potential conflict of interest relevant to this article was reported.

\section{REFERENCES}

1. Aldrete JA, Ghaly R: Postlaminectomy pseudomeningocele. An unsuspected cause of low back pain. Reg Anesth 20:75-79, 1995

2. Black P: Cerebrospinal fluid leaks following spinal surgery: use of fat grafts for prevention and repair. Technical note. J Neurosurg 96(2 Suppl):250-252, 2002

3. Bosacco SJ, Gardner MJ, Guille JT: Evaluation and treatment of dural tears in lumbar spine surgery: a review. Clin Orthop Relat Res (389):238-247, 2001

4. Cammisa FP Jr, Girardi FP, Sangani PK, Parvataneni HK, Cadag S, Sandhu HS: Incidental durotomy in spine surgery. Spine (Phila Pa 1976) 25:2663-2667, 2000

5. DiFazio FA, Nichols JB, Pope MH, Frymoyer JW: The use of expanded polytetrafluoroethylene as an interpositional membrane after lumbar laminectomy. Spine (Phila Pa 1976) 20:986991, 1995
6. Eismont FJ, Wiesel SW, Rothman RH: Treatment of dural tears associated with spinal surgery. J Bone Joint Surg Am 63:11321136, 1981

7. Espiritu MT, Rhyne A, Darden BV 2nd: Dural tears in spine surgery. J Am Acad Orthop Surg 18:537-545, 2010

8. Hadani M, Findler G, Knoler N, Tadmor R, Sahar A, Shacked I: Entrapped lumbar nerve root in pseudomeningocele after laminectomy: report of three cases. Neurosurgery 19:405-407, 1986

9. Hida K, Yamaguchi S, Seki T, Yano S, Akino M, Terasaka S, et al: Nonsuture dural repair using polyglycolic acid mesh and fibrin glue: clinical application to spinal surgery. Surg Neurol 65:136-142, 2006

10. Hodges SD, Humphreys SC, Eck JC, Covington LA: Management of incidental durotomy without mandatory bed rest. A retrospective review of 20 cases. Spine (Phila Pa 1976) 24:20622064, 1999

11. Khan MH, Rihn J, Steele G, Davis R, Donaldson WF 3rd, Kang JD, et al: Postoperative management protocol for incidental dural tears during degenerative lumbar spine surgery: a review of 3,183 consecutive degenerative lumbar cases. Spine (Phila Pa 1976) 31:2609-2613, 2006

12. Koo J, Adamson R, Wagner FC Jr, Hrdy DB: A new cause of chronic meningitis: infected lumbar pseudomeningocele. Am J Med 86:103-104, 1989

13. Mayfield FH, Kurokawa K: Watertight closure of spinal dura mater. Technical note. J Neurosurg 43:639-640, 1975

14. Mesfin A, McCarthy EF, Kebaish KM: Surgical treatment of aneurysmal bone cysts of the spine. Iowa Orthop J 32:40-45, 2012

15. Jeong SK, Seong HY, Roh SW: Extra-intradural Spinal Meningioma: a case report. Korean J Spine 11:202-204, 2014

16. Narotam PK, José S, Nathoo N, Taylon C, Vora Y: Collagen matrix (DuraGen) in dural repair: analysis of a new modified technique. Spine (Phila Pa 1976) 29:2861-2867, 2004

17. O'Connor D, Maskery N, Griffiths WE: Pseudomeningocele nerve root entrapment after lumbar discectomy. Spine (Phila Pa 1976) 23:1501-1502, 1998

18. Parízek J, Měricka P, Husek Z, Suba P, Spacek J, Němecek S, et al: Detailed evaluation of 2959 allogeneic and xenogeneic dense connective tissue grafts (fascia lata, pericardium, and dura mater) used in the course of 20 years for duraplasty in neurosurgery. Acta Neurochir (Wien) 139:827-838, 1997

19. San-Galli F, Deminière C, Guérin J, Rabaud M: Use of a biodegradable elastin-fibrin material, Neuroplast, as a dural substitute. Biomaterials 17:1081-1085, 1996

20. Wang JC, Bohlman HH, Riew KD: Dural tears secondary to operations on the lumbar spine. Management and results after a two-year-minimum follow-up of eighty-eight patients. J Bone Joint Surg Am 80:1728-1732, 1998 